Results $10 \%$ of prior relapsers and $31-40 \%$ of partial responders (shaded cells), had $<1 \log 10$ decline in HCV RNA at week 4 in the control and L-I arm, respectively. SVR rates in the T L-I arm among prior relapsers and partial responders were higher $(62 \%$ and $56 \%$, respectively; combined $\mathrm{SVR}=58 \%$ ) than in prior week $12 \mathrm{NR}$ who experienced $<1 \log 10$ decline in HCV RNA (15\%). Although patients with $-1 \log 10$ response at end of the L-I phase had the highest SVR rates, SVR in T/PR patients with $<1 \log 10$ was considerably higher $(62 \pm 15 \%)$ than control $(0 \%)$.

Conclusion Poor interferon responders on treatment $(<1 \log 10$ decline in HCV RNA at week 4) are not the same as prior PR NR $(<2 \log 10$ at week 12$)$. SVR rates in T/PR patients were higher than control irrespective of their response $(<$ or $-1 \log 10)$ at the end of the L-I phase. Safety findings in the T arm were similar irrespective of week 4 response.

\section{P60 PRE-TREATMENT IP-10 CONCENTRATIONS ARE ASSOCIATED WITH A SUSTAINED VIROLOGICAL RESPONSE IN HIV/HCV CO-INFECTED PATIENTS}

doi:10.1136/gutjnl-2011-300857a.60

D Joshi, M Bruce, A Suddle, C Taylor, I Carey, K Agarwal. King's College Hospital

Introduction Treatment of chronic HCV (cHCV) in HIV positive patients with pegylated interferon (PEG-IFN) and ribavirin (RIB) is associated with poorer treatment outcomes and an increased side effect profile. Strong immune $\mathrm{T}$ helper type 1 responses towards $\mathrm{HCV}$ determine outcome of infection. Interferon $\gamma$ inducible protein 10 (IP-10) has been shown to correlate with treatment response in cHCV mono-infection but limited data are available for HIV co-infected patients.

Aim To investigate whether pre-treatment plasma levels of cytokines/chemokines differ between HIV/HCV co-infected and HCV mono-infected patients and whether they can predict response to PEG-IFN and RIB therapy.

Method Pre-treatment plasma samples were studied in HIV positive patients co-infected with $\mathrm{CHCV}$ and HIV negative $\mathrm{CHCV}$ patients. Plasma levels of IFN- $\boldsymbol{\gamma}$, interleukin (IL)-2, IL-4, IL-6, IL-8, IL-10 and IP-10 (all pg/ml) were measured by ELISA. Patients were matched according to genotype, fibrosis stage and age. All patients were treated with PEG-IFN $\alpha$ 2a and weight based RIB for 24 weeks or 48 weeks according to genotype. All patients were previously treatment naive. Virological response was divided into SVR, nonresponse (NR) and responder relapse (RR). All results are presented as medians.

Results 37 HIV positive patients (CD4 count 495 cells/ml, undetectable HIV viral load in $86 \%$ ) co-infected with HCV ([HCV viral load (VL) 1.68E6 IU/ml, 54\% genotype 1, 7 (19\%) patients with advanced fibrosis on biopsy (Ishak $\mathrm{F}>5$ )] were divided according virological response [ $\mathrm{n}=23$ SVR (62\%), $n=7 \mathrm{RR}, \mathrm{n}=7 \mathrm{NR}$ ]. $35 \mathrm{HIV}$ negative patients with cHCV [HCV VL 2.12E6, 64\% genotype 1, 5 (14\%) patients with advanced fibrosis (Ishak F>5) were also divided according to virological response $[n=23 \operatorname{SVR}(66 \%), n=7 R R, n=6$ NR]. Irrespective of HIV status, IP-10 (62 vs $105, \mathrm{p}=0.006$ ) and IL-6 (4 vs 7, $\mathrm{p}=0.03$ ) levels were lower at baseline in patients who achieved an SVR. Baseline IP-10 (92 vs 49, $\mathrm{p}=0.009$ ) and IL-8 (39 vs 22, $\mathrm{p}=0.01$ ) were higher in the HIV positive group but the concentrations of IFN- $\gamma$, IL-2, IL-4, IL-6, IL-10 were similar between the two groups. In HIV positive patients baseline IP-10 concentrations were lower in those who achieved a SVR (84 vs $107 \mathrm{p}=0.02$ ) and also differed according to treatment response (SVR, 84 vs RR, 124 vs NR, $107 \mathrm{p}=0.03$ ).

Conclusion In our cohort IP-10 acted as a robust predictor of SVR among HIV/HCV co-infected and HCV mono-infected patients. IP-10 could serve as a pre-treatment biomarker to help identify patients who will achieve a SVR.

\section{P61 BONE MINERAL DENSITY LOSS IN TENOFOVIR TREATED CHRONIC HEPATITIS B VIRUS (HBV) PATIENTS IS A CONSEQUENCE OF VITAMIN D DEFICIENCY AND NOT TENOFOVIR THERAPY}

doi:10.1136/gutjnl-2011-300857a.61

${ }^{1}$ U S Gill, ${ }^{1} \mathrm{~S}$ Al-shamma, ${ }^{1} \mathrm{~K}$ Burke, ${ }^{2} \mathrm{~V}$ Ross, ${ }^{1} \mathrm{R}$ T C Marley, ${ }^{1} \mathrm{P}$ Kooner, ${ }^{1} \mathrm{G}$ R Foster, ${ }^{1} \mathrm{P}$ T F Kennedy. ${ }^{1}$ Department of Hepatology, Barts and The London NHS Trust; ${ }^{2}$ Pharmacy, Barts and The London NHS Trust

Introduction Tenofovir Disoproxil Fumarate (TDF) is now established as a very potent oral antiviral (OAV) agent in the treatment of chronic hepatitis B (CHB). However, as treatment with this OAV is often indefinite and potentially lifelong, concerns remain about its long-term safety. Bone Mineral Density (BMD) loss has been described in TDF treated HIV patients, but limited data exist for HBV treated patients. Furthermore, BMD loss has also been described in chronic liver disease in addition to being reported with certain patient characteristics.

Aim The aim of this study was to determine the impact of TDF on $\mathrm{BMD}$ in an ethnically diverse HBV infected population undergoing long-term treatment with this agent.

Method CHB patients treated with TDF for a minimum of 12 months were recruited to this single centre study. Patients were prospectively offered a dual x-ray absorptiometry (DEXA) scan. Serum bone profile and Vitamin D levels were requested simultaneously. BMD loss was defined by WHO criteria; T-score $<-2.5$ (osteoporosis) and between -1 and -2.5 (osteopenia). 107 consecutive TDF treated patients were included (78 males), median age 45 (range 26-64). A control group, $27 \mathrm{CHB}$ patients (19 males), median age 32 (range 20-61), with no TDF exposure were also studied. Data on gender, ethnicity, BMI, fibrosis stage, co-morbidities and drug history were recorded in all subjects. Analysis was performed with SPSS V.19.

Results $\mathrm{BMD}$ loss was present in $44 \%$ of the treatment group (osteopenia $81 \%$, osteoporosis 19\%) and in $44 \%$ of the control group (osteopenia $83 \%$, osteoporosis $17 \%$ ) ( $p=0.21$ ). In the ethnically diverse population studied, there was more marked BMD loss in the non-white population ( $47 \%$, treated group; $45 \%$, controls) compared with the white population (30\%, treated group; $40 \%$, controls). By univariate analysis age, gender, ethnicity, fibrosis stage, BMI, comorbidities and low Vitamin D level were all significant for reduced $\mathrm{BMD}$ ( $\mathrm{p} \leq 0.05$, all variables). On multivariate analysis gender, ethnicity, BMI, fibrosis, co-morbidities and Vitamin D all met statistical significance for a reduction in $\mathrm{BMD}$, but Vitamin $\mathrm{D}$ deficiency only was significant for the presence of osteoporosis $(\mathrm{p}=0.0001)$

Conclusion Our results demonstrate the prevalence of reduced BMD in CHB patients of diverse ethnicity and identify Vitamin D deficiency and not TDF as the likely cause. This cross-sectional study does not exclude the potential for BMD loss with TDF and further longitudinal studies are required to determine its effect on $\mathrm{BMD}$ over time. Vitamin D deficiency should be appropriately treated to avoid any potential for BMD loss associated with TDF when considering treatment with this OAV agent. 